\title{
Rhabdomyosarcoma of the Oral Cavity: A Case Report
}

\author{
Ozkan Miloglua \\ Sare Sipal Altas ${ }^{b}$ \\ Mustafa Cemil Buyukkurtc \\ Burak Erdemcid \\ Oguzhan Altun ${ }^{\mathrm{e}}$
}

\section{ABSTRACT}

Rhabdomyosarcoma (RMS), a tumor of skeletal muscle origin, is the most common soft tissue sarcoma encountered in childhood and adolescence. The common sites of occurrence are the head and neck region, genitourinary tract, retroperitonium, and, to a lesser extent, the extremities. In the head and neck region, the most commonly affected sites are the orbit, paranasal sinuses, soft tissues of the cheek, and the neck. RMS is relatively uncommon in the oral cavity, and the involvement of the jaws is extremely rare. Here, we report a case of oral RMS in a 13-year-old child and describe the clinical, radiological, histopathological, and immunohistochemical findings. (Eur J Dent $2011 ; 5: 340-3431$

Key words: Mouth neoplasm; Oral pathology; Alveolar rhabdomyosarcoma; Radiotherapy; Chemotherapy.

\section{INTRODUCTION}

Rhabdomyosarcoma (RMS), which was first described by Weber in 1854, is a malignant soft

a Department of Oral Diagnosis and Radiology, Faculty of Dentistry, Ataturk University, Erzurum, Turkey.

b Department of Pathology, Faculty of Medicine, Ataturk University, Erzurum, Turkey.

c Department of Oral and Maxillofacial Surgery, Faculty of Dentistry, Sifa University, Izmir, Turkey.

d Department of Radiation Oncology, Faculty of Medicine, Ataturk University, Erzurum, Turkey.

e Department of Oral Diagnosis and Radiology, Faculty of Dentistry, Inonu University, Malatya, Turkey.

- Corresponding author: Ozkan Miloglu Department of Oral Diagnosis and Radiology, Faculty of Dentistry,

Ataturk University, Erzurum, Turkey.

Phone: +904422311805

Fax: +904422360945

E-mail: omilogluahotmail.com tissue neoplasm of skeletal muscle origin. It accounts for $6 \%$ of all malignancies in children under 15 years of age. ${ }^{1}$ The most commonly affected areas are the head and neck region, genitourinary tract, retroperitonium, and, to a lesser extent, the extremities. ${ }^{2}$ The head and neck RMSs are anatomically divided into 2 categories: parameningeal lincluding RMS of the nose, nasopharynx, paranasal sinuses, middle ear, mastoid, infratemporal fossa, and pterygopalatine fossal and nonparameningeal lincluding RMS of the scalp, orbit, parotid gland, oral cavity, oropharynx, and larynx). RMS of the oral cavity accounts for $10-12 \%$ of all the head and neck RMS cases. ${ }^{3}$

Clinically, the manifestations of RMS may vary from a small cutaneous nodule on the face to an extensive fast-growing facial swelling, which may 
be painless or occasionally associated with pain, trismus, paresthesia, facial palsy, and nasal discharge. ${ }^{1,4}$

On the basis of the histological findings, 4 broad subtypes of RMS have been identified: botryoid and spindle cell RMS, embryonal RMS, alveolar RMS, and undifferentiated RMS.5,6 The histogenesis of RMS is still unclear, but the most widely accepted hypothesis is that RMS arises due to proliferation of embryonic mesenchymal tissue. ${ }^{7}$

In this report, we present a case of oral RMS arising within the left cheek mucosa and involving the mandibular bone in a 13-year-old girl, and we describe the clinical, radiological, histopathological, and immunohistochemical features of this RMS.

\section{CASE REPORT}

A 13-year old girl was referred to our institution in August 2006 for the investigation of painful swelling in her mouth. On history taking, her parents reported that the disease had started as a painless swelling in May 2006, and that she had received oral and parenteral antibiotics for the past 4 months; however, the treatments were ineffective, and she was therefore referred to us. Written informed consent was obtained from her parent for further investigations.

Clinical examination showed severe facial asymmetry. A large, firm, and tender swelling was noted on the left cheek and it caused pain. The skin had appeared stretched and inflamed. The opening of the mouth was partly restricted. Intraoral examination revealed an extensive mass involving the buccal mucosa (Figure 1). Magnetic resonance imaging (MRI) showed an infiltrative large soft tissue lesion in the maxillary sinus, infraorbitary space, pterygopalatine fossa, pterygoid plates, and the ramus, which caused displacement of the adjacent structures (Figure 2). An incisional biopsy was made. Histological examination of the specimen showed clusters of small round cells with hyper chromatic nuclei and eosinophilic cytoplasm separated by fibrovascular septae (Figure 3). The morphologic features of the cells indicated a differential diagnosis comprising RMS, Ewing's sarcoma, malignant melanoma, and an epithelial tumor. The neoplastic cells were strongly positive for vimentin, desmin, myoglobin, and muscle specific-actin (Figure 4) and negative for CD99, chromogranin, S 100, HMB 45, EMA, and Pan CK. After performing the standard diagnostic work-up, the tumor was diagnosed as alveolar RMS. She was referred to the Pediatric Oncology Department, and the proposed treatment plan was a combination of chemotherapy, including ifo sfamid+vincristine+actinomycine-D (IVA regimen) and Mesna, and radiotherapy. Although the tumor showed dramatic regression after the initial 12 weeks of chemotherapy, the patient's parents refused external beam radiation therapy. During the post-chemotherapy radiographic evaluation, the tumor was found to have regressed. However, after 6 months, she experienced an increase in pain and mass localization, limitations in temporomandibular articulation, and loss in weight; therefore, she reconsulted the Oncology Clinic. Surprisingly, the tumor was found to have rapidly increased in size, almost reaching the pretreatment size, indicating the reversal of the effects of chemotherapy. Owing to this development, she was administered chemotherapy with carboplatin+epirubisin+vincris tine for 8 weeks. Following the completion of chemotherapy, she was treated with external beam radiation therapy $(5,400 \mathrm{cGY})$ for 8 weeks. Despite the treatment, the tumor continued to increase in size, and she died 2 years after the initial treatment.

\section{DISCUSSION}

After neuroblastoma and Wilms tumor, RMS is the third most common extracranial malignant tumor that affects children. Of all soft tissue sarcomas, RMS accounts for only $2-5 \%$ tumors in adults but approximately $60 \%$ tumors in children. 8 The incidence of RMS is highest in children aged 1-4 years, lower in children aged 10-14 years, and lowest in those aged 15-19 years. 5,6 Our patient was 13 years old and did not fall in the very highrisk category.

The head and neck region is the most common site for RMS, with the orbit being the most frequent primary site. The most common site of involvement in the oral cavity is the tongue followed by the soft palate, hard palate, and buccal mucosa. ${ }^{9}$ In our patient, the buccal mucosa, mandibular alveolar ridges, and hard palate were all involved. The soft tissue was more damaged than the bone, suggesting that the tumor primarily originated in the buccal mucosa. 
In general, most patients have an advanced disease even at the stage of initial presentation because RMSs are known to show rapid growth and the patients generally tend to delay medical consultation..$^{5,6}$ In our case, when the patient presented at our clinic, she had severe facial asymmetry and experienced difficulty in opening the mouth. In cases of RMS, radiographic examination reveals the size of the lesion, space relation, and the extent of bone destruction, while MRI reveals the affected areas such as the mandible, condyle, maxillary sinus, and infra-orbitary space.

A careful histological examination is required to differentiate such lesions from other more frequent and aggressive lesions affecting the concerned site. In our case, the marked pleomor-

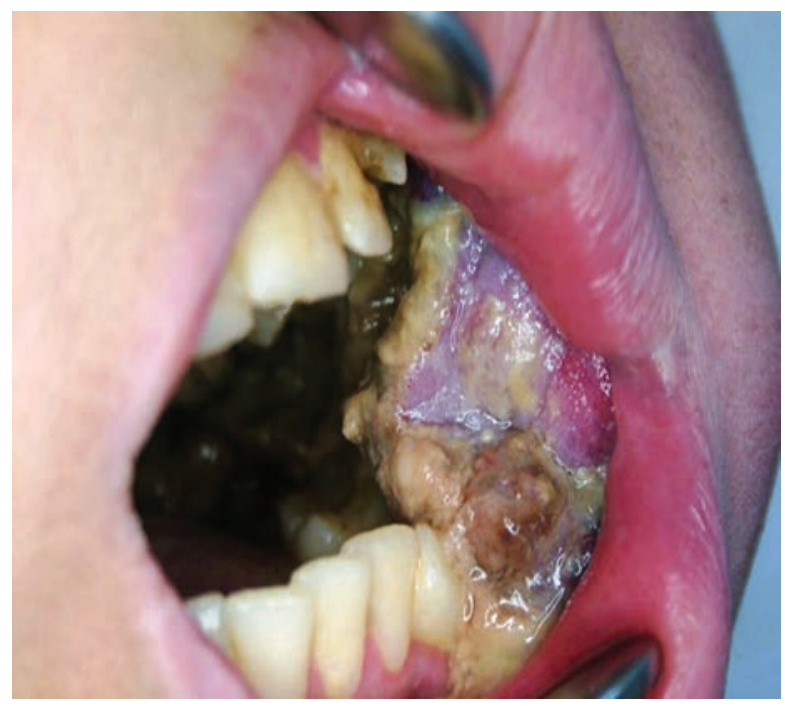

Figure 1. Intraoral aspect showing extensive mass involving the buccal mucosa.

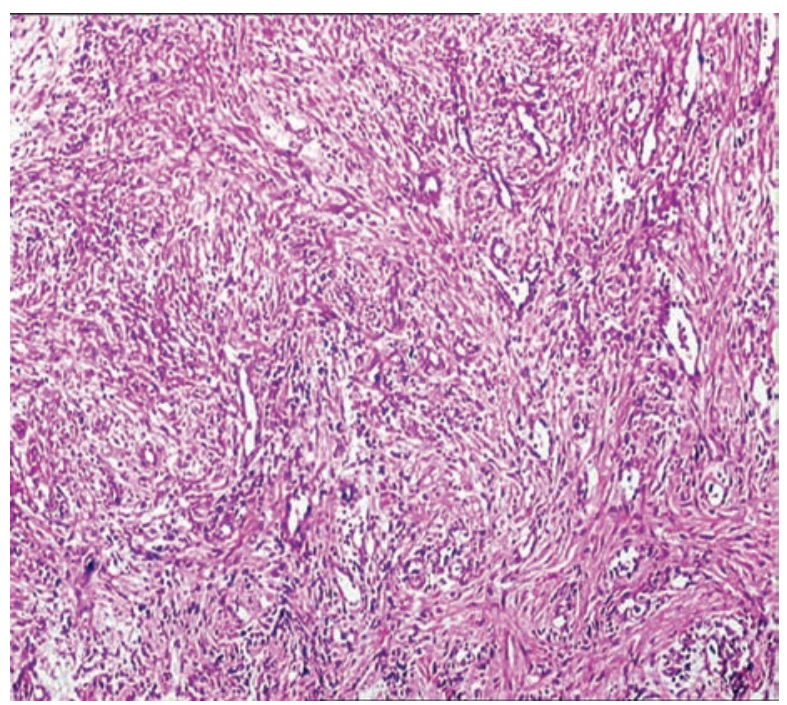

Figure 3. Photomicrograph showing a sheet of mesenchymal cells in a myxoid stroma (Hemotoxylin/Eosin (H/E) x200). phism noted was critical for differentiating RMS from Ewing's sarcoma. The presence of an alveolar pattern, pleomorphism, cohesive nature of the cells, and the absence of lymphadenopathy ruled out the diagnosis of lymphoma. In this respect, the most critical differential diagnosis is neuroblastoma. A diffuse pattern of small round cells and the presence of rosettes/pseudorosettes with pale eosinophilic material seen in cases of neuroblastoma may suggest alveolar RMS. High level of urinary catecholamines observed in neuroblastoma is critical to rule out this tumor. 8,9

Prognosis of RMS is relatively poor compared to that of other oral soft tissue malignant lesions ${ }^{10,11}$ and depends on the clinical staging and the anatomic site of the tumor. ${ }^{10}$ An early and accurate

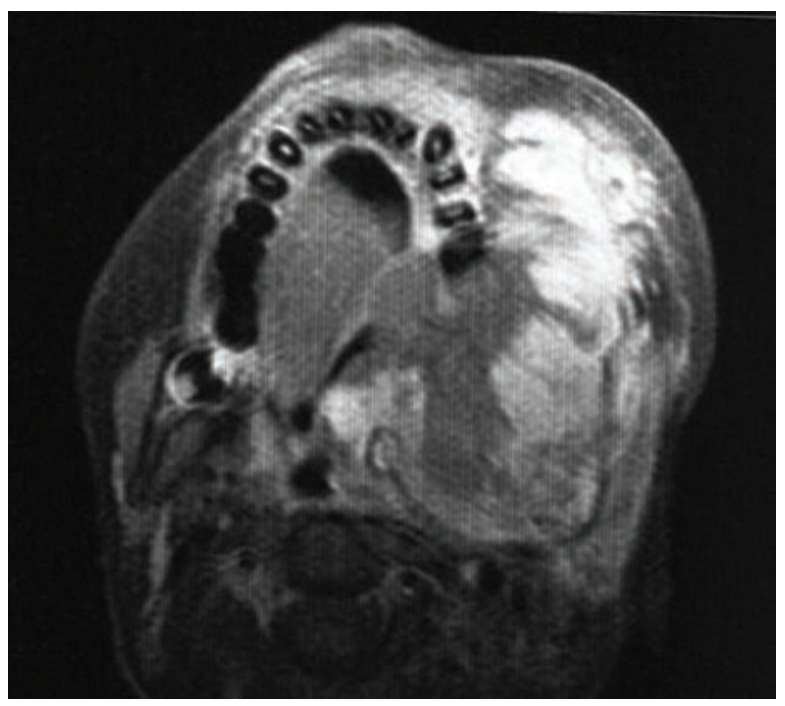

Figure 2. Magnetic Resonance Imaging (MRI) scan showing an infiltrative large soft tissue lesion in the maxillary sinus, infra-orbitary space, pterygopalatine fossa, pterygoid plates, and the ramus causing displacement of the adjacent structures.

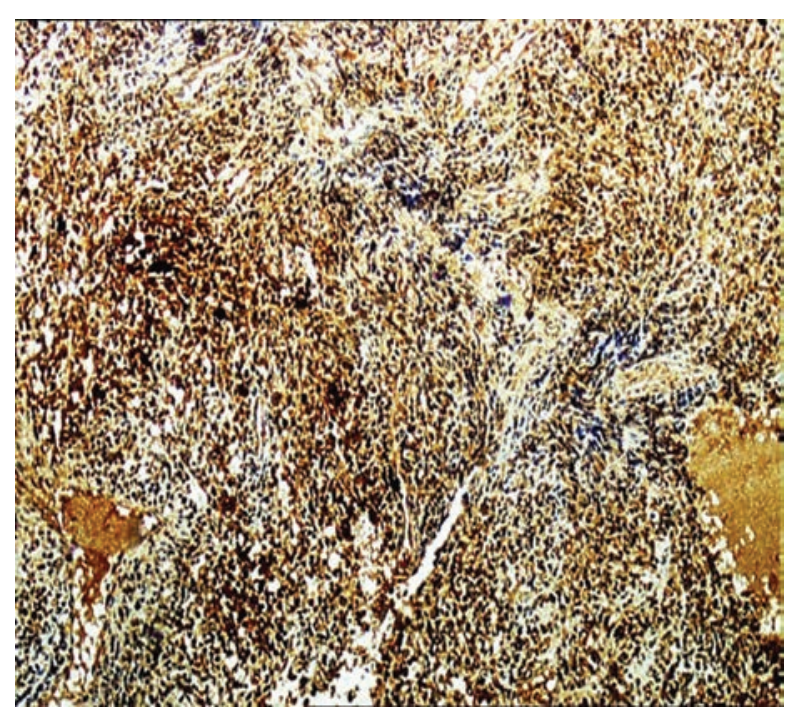

Figure 4. Positive immunohistochemical staining for muscle specific-actin (streptavidin biotin $\times 200$ ). 
diagnosis of the tumor and a combined therapeutic approach involving surgery, chemotherapy, and radiation therapy are known to dramatically improve the survival rates, as seen in cases recorded over the past 20 years. ${ }^{8}$ Effective surgical excision is challenging in cases of RMS of the head or neck region owing to the involvement of other crucial structures in these locations. Therefore, we planned a multidisciplinary approach with induction chemotherapy and radiotherapy to bring about tumor regression in our patient. Although the tumor showed a dramatic shrinkage after the initial 12 weeks of chemotherapy, the patient's parents refused external beam radiation therapy. However, 6 months later, when the patient reconsulted at the Oncology Department for a recurrent lesion, she was administered further chemotherapy and radiation therapy. Unfortunately, the tumor increased in size despite the treatments, and she died 2 years after the initial treatment.

Although a marked improvement in the survival rates has been reported in patients with RMS in recent years, a poor prognosis in patients $>10$ years old, delay diagnosis, and lack of cooperation from the patient and the family are reported to have a negative impact on the prognosis, as in our case.

Dental practitioners frequently encounter cases of children with facial swelling and pain; in most of these cases, dental abscess is considered as the cause and treatment is prescribed accordingly. Taken together, we conclude that in children, any swelling should be carefully examined and treatment outcomes should be regularly followed up. High degree of suspicion, early diagnosis, and a multidisciplinary treatment approach would be of great importance in such cases.

\section{ACKNOWLEDGEMENT}

We would like to express our sincere gratitude to Lecturer Ali Çaḡlar Güllüce for his help in proofreading our article.

\section{REFERENCES}

1. Chigurupati R, Alfatooni A, Myall RW, Hawkins D, Oda D. Orofacial rhabdomyosarcoma in neonates and young children: a review of literature and management of four cases. Oral Oncol 2002;8:508-515.
2. Durães GV, Jham BC, Mesquita ATM, dos Santos CRR, Miranda JL. Oral embryonal rhabdomyosarcoma in a Child: A case report with immunohistochemical analysis. Oral Oncol Extra 2006;42:105-108.

3. Al-Khateeb T, Bataineh AB. Rhabdomyosarcoma of the oral and maxillofacial region in Jordanians: a retrospective analysis. Oral Surg Oral Med Oral Pathol Oral Radiol Endod 2002;93:580-585

4. Wiss K, Solomon AR, Raimer SS, Lobe TE, Gourley W, Headington JT. Rhabdomyosarcoma presenting as a cutaneous nodule. Arch Dermatol 1988;124:1687-1690.

5. França CM, Caran EM, Alves MT, Barreto AD, Lopes NN. Rhabdomyosarcoma of the oral tissues-two new cases and literature review. Med Oral Patol Oral Cir Bucal 2006;11:136-140.

6. Gordón-Núñez MA, Piva MR, Dos Anjos ED, Freitas RA. Orofacial rhabdomyosarcoma: report of a case and review of the literature. Med Oral Patol Oral Cir Bucal 2008;13:765769.

7. Loducca SV, Mantesso A, de Oliveira EM, de Araújo VC. Intraosseous rhabdomyosarcoma of the mandible: A case report. Int J Surg Pathol 2003;11:57-60.

8. Sekhar MS, Desai S, Kumar GS. Alveolar rhabdomyosarcoma involving the jaws: a case report. J Oral Maxillofac Surg 2000;58:1062-1065.

9. Hicks J, Flaitz C. Rhabdomyosarcoma of the head and neck in children. Oral Oncol 2002;38:450-459.

10. Peters E, Cohen M, Altini M, Murray J. Rhabdomyosarcoma of the oral and paraoral region. Cancer 1989;63:963-966.

11. Yamaguchi S, Nagasawa H, Suzuki T, Fujii E, Iwaki H, Takagi $M$, et al. Sarcomas of the oral and maxillofacial region: a review of 32 cases in 25 years. Clin Oral Investig 2004;8:5255. 